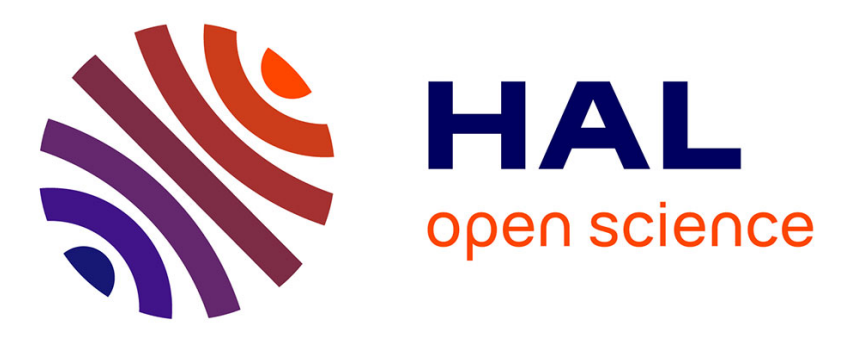

\title{
Magnetic field induced crossover in weakly localized regimes and scaling of the conductivity
}

R. Oppermann

\section{To cite this version:}

R. Oppermann. Magnetic field induced crossover in weakly localized regimes and scaling of the conductivity. Journal de Physique Lettres, 1984, 45 (24), pp.1161-1165. 10.1051/jphyslet:0198400450240116100 . jpa-00232464

\section{HAL Id: jpa-00232464 https://hal.science/jpa-00232464}

Submitted on 1 Jan 1984

HAL is a multi-disciplinary open access archive for the deposit and dissemination of scientific research documents, whether they are published or not. The documents may come from teaching and research institutions in France or abroad, or from public or private research centers.
L'archive ouverte pluridisciplinaire HAL, est destinée au dépôt et à la diffusion de documents scientifiques de niveau recherche, publiés ou non, émanant des établissements d'enseignement et de recherche français ou étrangers, des laboratoires publics ou privés. 


\title{
Magnetic field induced crossover in weakly localized regimes and scaling of the conductivity
}

\section{R. Oppermann}

Institut für Theoretische Physik, Ruprecht-Karls-Universität, D-6900 Heidelberg, F.R.G.

(Reçu le 27 août 1984, révisé le 29 octobre, accepté le 30 octobre 1984)

\begin{abstract}
Résumé. - La conductivité pour un ensemble matriciel complexe représentant un système désordonné bidimensionnel (2D) dans un champ magnétique perpendiculaire $H_{\perp}$ est calculée comme une fonction de la longueur, de la fréquence, du paramètre de crossover $x\left(H_{\perp}\right)$ et de la conductance nue $g_{0}$ jusqu'à $O\left(g_{0}^{-2}\right)$. Au-dessus de $2 \mathrm{D}$ les expressions biparamétriques $\sigma \propto \Delta_{1} f_{1}\left((1-x) / \Delta_{1}^{\varphi_{1}}\right)$ $\operatorname{avec} \Delta_{x} \equiv\left|E_{\mathrm{F}}-E_{\mathrm{ME}}(x)\right| / E_{\mathrm{ME}}(x), 0 \lesssim 1-x \propto H_{\perp}$ près de la limite orthogonale et $\sigma \propto \sqrt{\Delta_{0}} f_{0}\left(x^{2} / \Delta_{0}^{\varphi_{0}}\right)$ avec l'amplitude de Cooper $x\left(H_{\perp}\right)$ près de la limite invariante de phases donnent les exposants de crossover $\varphi_{1}=2 v_{1}=2 /(D-2)$ et $\varphi_{0}=0 / \sqrt{D-2}+\mathcal{O}\left((D-2)^{0}\right)$.

Abstract. - For a complex matrix ensemble modelling a two-dimensional (2D) disordered system in a perpendicular magnetic field $H_{\perp}$ the conductivity is calculated as a function of length, frequency, crossover parameter $x\left(H_{\perp}\right)$, and bare conductance $g_{0}$ up to $\mathcal{O}\left(g_{0}^{-2}\right)$. Above 2D the scaling forms $\sigma \propto \Delta_{1} f_{1}\left((1-x) / \Delta_{1}^{\varphi_{1}}\right)$ with $\Delta_{x} \equiv\left|E_{\mathrm{F}}-E_{\mathrm{ME}}(x)\right| / E_{\mathrm{ME}}(x), 0 \lesssim 1-x \propto H_{\perp}$ near the orthogonal limit and $\sigma \propto \sqrt{\Delta_{0}} f_{0}\left(x^{2} / \Delta_{0}^{\varphi_{0}}\right)$ with cooperon amplitude $x\left(H_{\perp}\right)$ near the phase invariant limit yield the crossover exponents $\varphi_{1}=2 v_{1}=2 /(D-2)$ and $\varphi_{0}=0 / \sqrt{D-2}+\mathcal{O}\left((D-2)^{0}\right)$.
\end{abstract}

A magnetic field introduces many complications into the theory of localization of noninteracting electrons in disordered systems. There are symmetry violations like breaking of time reversal and translational invariance, spatial isotropy is lost in general, and quantitative effects like electronic wavefunction shrinkage with increasing field strength occur. Moreover, surface states and topological excitations seem to play an important role [1-3]. Possibly comparable difficulties are known in the theory of superconductors, e.g. vortex excitations and the mixed phase in dirty superconductors [4].

This paper reports detailed results for the conductivity of a two-dimensional system in a perpendicular magnetic field. Multiplicative renormalization is used to derive the crossover scaling behaviour near the zero-field and near the "strong field" fixed point above two dimensions. For this purpose the complex matrix ensemble is used, a model which takes care of the breaking of time reversal invariance and also includes the effect of wavefunction shrinkage.

For variable magnetic field strength only incomplete details on logarithmic quantum corrections of the $2 \mathrm{D}$ conductivity in the weakly localized regime were known so far. These are formally summarized by

$$
\sigma(\Omega)=\sigma_{0}\left(1+\left(\frac{s e^{2} / \hbar}{4 \pi^{2} \sigma_{0}}\right)^{\alpha} \ln (\Omega \tau)+\mathcal{O}\left(\sigma_{0}^{-2-2 \alpha}\right)\right),
$$


where $\alpha=0$ is the zero-field case [5,6], while the " strong field » $\alpha=1$ solution has been obtained both by phase factor approximation with an $n$-orbital model [7] and by the opposite modelling of zeroth Landau level occupation only [8,9]. For weak fields the negative $\ln H$-magnetoresistance and the $\log T$-temperature behaviour due to inelastic scattering $[10,11]$ are most important, and these are big effects due to the sensitivity of the cooperon. However, for increasing magnetic field strength the critical region where localization is due to diffusons instead of cooperons becomes broader. Crossover behaviour is very interesting in the weakly localized regime, since both arguments and amplitudes of the logarithmic quantum corrections become dependent on the magnetic length $l_{H}=\sqrt{\hbar c / e H}$.

Instead of the formal discrete parameter $\alpha=0,1$ the complex matrix ensemble offers a continuous crossover parameter $1 \geqslant x>-1$, where for $l_{H}$ sufficiently larger than the diffusive length $l=2 \sqrt{D_{0} \tau}$.

$$
x=1-l^{2} / l_{H}^{2} .
$$

In terms of earlier publications [7], $x=M_{\mathrm{pp}} / M_{\mathrm{ph}}$ denotes the ratio between particle-particle and particle-hole scattering amplitude. For $2 \mathrm{D}$ and $H_{\perp}$, all divergent conductivity corrections depending on frequency $\Omega$, length $L$, on disorder via the bare conductance $g_{0}$, and on magnetic field through $x$ are contained in

$$
\begin{aligned}
& \frac{\sigma(L, \Omega, x)}{\sigma_{0}}=1+\frac{\operatorname{Re}}{g_{0}}\left\{\frac{1}{x} \ln \left(\frac{1-x+l^{2} \lambda^{2}}{1+x+l^{2} \lambda^{2}}\right)+2\right\} \\
& +\frac{\operatorname{Re}}{g_{0}^{2}}\left\{\left(1-\frac{l^{2} \lambda_{\Omega}^{2} x}{1-x+l^{2} \lambda^{2}}\right) \ln \left(l^{2} \lambda^{2}\right)\right. \\
& \left.-\ln \left(\frac{1-x+l^{2} \lambda^{2}}{1+x+l^{2} \lambda^{2}}\right)+\mathcal{O}\left((1-x) \ln ^{2}\left(\frac{1-x}{1+x}\right)\right)\right\},
\end{aligned}
$$

where $\lambda^{2} \equiv \lambda_{\Omega}^{2}+\lambda_{L}^{2} \equiv-i \Omega / D_{0}+(2 \pi / L)^{2}, g_{0}=4 \pi^{2} \sigma_{0} \lambda_{L}^{2-D}$, and Re means the real part. The four diagram classes participating in the two-loop contribution given are shown in table I together with their values. The accuracy of formula (3) needs the following explanations :

i) small corrections of order $\lambda^{2} \ln \lambda^{2}$ are not given in (3), but the dangerous last term in (3) changes order and must be kept. Its amplitude $\lambda_{\Omega}^{2} / \lambda^{2}$ is exact in the $x \rightarrow 1\left(l_{H} \rightarrow \infty\right)$ limit and all divergences in the phase invariant limit with $x \rightarrow 0$ are cancelled in (3). The sum of all $\lambda_{\Omega}^{2} \ln \left(l^{2} \lambda^{2}\right)$ contributions given in (3) vanishes like $x$ for $x \rightarrow 0$. The full result vanishes like $x^{2}$ for $x \rightarrow 0$.

ii) terms of order $\ln ^{a}\left(\frac{1-x}{1+x}\right)$ are kept exactly for $a=2$, 1 , while finite contributions of order $(1-x) \ln ^{a}\left(\frac{1-x}{1+x}\right)$ are irrelevant.

iii) the region of validity is limited by $x+1 \gg l^{2} \lambda^{2}$, since singular density of states effects emerge near $x=-1$. Density of states effects are eliminated by the band centre calculation of (3). Apart from these minor approximations, formula (3) covers exactly the entire crossover region. It continuously links the $2 \mathrm{D}$ results for two ensembles of different symmetry. Above $2 \mathrm{D}$ it can moreover be used in a $D-2$ expansion in order to relate the nonlinear $\sigma$-model results for orthogonal and unitary symmetry $[12,13]$ and to consider their stability [14].

An expansion of (3) around the unitary-phase-invariant solution yields

$$
\frac{g}{g_{0}}=1+g_{0}^{-2} \ln \left(l^{2} \lambda^{2}\right)-\frac{2}{3} x^{2} g_{0}^{-1}\left(1+\left(0 . g_{0}^{-1}+A g_{0}^{-2}\right) \ln \left(l^{2} \lambda^{2}\right)\right)+\mathcal{O}\left(x^{3}\right) \text {. }
$$


Table I. - Conductivity contribution of diagram classes described in reference [6]. Abbreviations used : $A_{x}=1-x+l^{2} \lambda^{2}, B_{x}=1+x+l^{2} \lambda^{2}$, and $A_{1}=A_{x=1} . k$ means the external momentum and taking the real part of all brackets is understood. The Cooper channel is marked by vectorlike propagators, while diffusions are scalars. Each line contains a full T-matrix and the broken lines are non-critical.

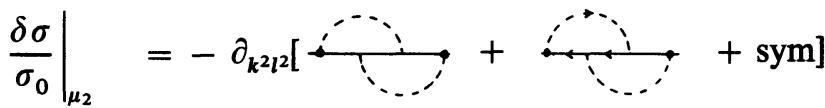

$$
\begin{aligned}
& =\frac{1}{g_{0}^{2}}\left[\frac{1}{2} \ln ^{2} \frac{A_{1}}{B_{1}}+\ln \frac{A_{1}}{B_{1}}+\frac{2 x^{2}}{B_{x}}\left(\ln ^{2} \frac{A_{x}}{B_{x}}+2 \ln \frac{A_{x}}{B_{x}}\right)\right] \\
& \left.\frac{\delta \sigma}{\sigma_{0}}\right|_{\mu_{3}}=-\partial_{k^{2} l^{2}} \\
& =\frac{1}{g_{0}^{2}}\left[\frac{1}{x} \ln \frac{A_{x}}{B_{x}} \ln \frac{A_{1}}{B_{x}}+2 \ln \frac{A_{1}}{B_{x}}-\frac{1}{2 x} \ln ^{2} \frac{A_{x}}{B_{x}}-\ln \frac{A_{x}}{B_{x}}\right] \\
& \left.\frac{\delta \sigma}{\sigma_{0}}\right|_{20 d}=-\partial_{k^{2} l^{2}} \\
& =-\frac{1}{g_{0}^{2}}\left[\frac{1}{x} \ln \frac{A_{x}}{B_{x}} \ln \frac{A_{1}}{B_{x}}+\left(2+x \frac{l^{2} \lambda_{\Omega}^{2}}{A_{x}}\right) \ln \frac{A_{1}}{B_{x}}+2 \ln \frac{A_{x}}{B_{x}}\right] \\
& \left.\frac{\delta \sigma}{\sigma_{0}}\right|_{l_{5}+v_{4}}=-\partial_{k^{2} l^{2}} \\
& =-\frac{1}{2 g_{0}^{2}}\left[\ln ^{2} \frac{A_{1}}{B_{1}}+x^{2} \ln ^{2} \frac{A_{x}}{B_{1}}+\mathcal{O}\left(A_{x} \ln \frac{A_{x}}{B_{x}}\right)\right]
\end{aligned}
$$

The yet to be calculated three-loop coefficient $A$ of the $x^{2}$-derivative of $g$ may well be different from zero, unlike the here-obtained total cancellation at two-loop order. For $x=0$ the next correction is of 4-loop order.

Near the orthogonal fixed point one finds by linearization in $1-x$

$$
\frac{g}{g_{0}}=1+\frac{1}{g_{0}} \ln \left(l^{2} \lambda_{L}^{2}\right)+\frac{1-x}{g_{0}}\left(\frac{1}{l^{2} \lambda_{L}^{2}}+\ln \left(l^{2} \lambda_{L}^{2}\right)+\cdots\right)+\mathcal{O}\left(\frac{1-x}{g_{0}^{2}} \frac{1}{l^{2} \lambda_{L}^{2}}\right) .
$$

We have chosen $\lambda_{\Omega}=0$ for simplicity and since the frequency is not needed for the analysis of crossover exponents as given below. The strange $(1-x) / \lambda_{I}^{2}$ term will find its natural explanation there. For nonzero frequency only equation $(5 a)$ is remarkably changed by a contribution depending on whether the $L \rightarrow \infty$ or $\Omega \rightarrow 0$ limit is taken first. Details on this special noncommuting limit property of the zero-field real matrix ensemble will be published elsewhere. While equation (5a) can be multiplicatively renormalized in $2 \mathrm{D}$ and serves for a derivation of the crossover exponent in $D>2$, the experimentally interesting weak field region is obtained for $1-x>$ $l^{2} \lambda^{2}$, where equation (3) yields

$$
\begin{aligned}
\frac{g}{g_{0}} \cong 1-\frac{1}{g_{0}}\left\{\frac{l_{H}^{2}}{l_{H}^{2}-l^{2}} \ln \left(l_{H}^{2} / l^{2}\right)+2\right\} & \\
& +\frac{\operatorname{Re}}{g_{0}^{2}}\left\{\left(1-\frac{\left|\lambda_{\Omega}^{4}\right|}{\left(l_{H}^{-2}+\lambda_{L}^{2}\right)^{2}+\left|\lambda_{\Omega}^{4}\right|}\right) \ln \left(l^{2} \lambda^{2}\right)+\ln \left(l_{H}^{2} / l^{2}\right)\right\} .
\end{aligned}
$$


Near the phase invariant solution, where localization is generated by the field resistant diffusons, the magnetoresistance contributions in equation (4) are weaker.

The phase invariance condition $x=0$ should however not be interpreted as $l_{H}=l$. Neither the model nor the fact, that rather the particle-particle amplitude instead of the magnetic field is renormalized near the phase invariant point, leads to the conclusion of a fixed point field.

Renormalization : by multiplicative renormalization [15] agreement had been achieved [14] with the orthogonal $(x=1)$ and unitary $(x=0)$ field theories. While it does not seem possible to renormalize the theory for arbitrary $x$, one can, however, renormalize in the vicinity of the fixed points $x=0,1$. In particular, the crossover exponents and hence the stability of the fixed points can be analysed. The additional term of order $\varepsilon / g_{0}$ in an $\varepsilon$-expansion above $2 \mathrm{D}$ is not needed for the following discussion.

In the small field region the two parameter scaling conductivity obeys

$$
\sigma \propto \Delta f_{1}\left(y / \Delta^{\varphi_{1}}\right), \quad y=l^{2} / l_{H}^{2}
$$

where $\Delta=\left|E_{\mathrm{F}}-E_{\mathrm{ME}}^{1}\right| / E_{\mathrm{ME}}^{1}$ measures the distance of the Fermi energy from the zero-field mobility edge, the dimensionless quantity $y \equiv 1-x$ is chosen to represent the magnetic field perturbation, and $\varphi_{1}$ is the crossover exponent defined at $y=0$. Taking the $y$-derivative of (3) we observe that only the one-loop part contributes in leading order such that

$$
\left.\left(\partial_{y} g\right)_{\lambda^{\prime}}\right|_{H=0}=\left.b^{-2}\left(\partial_{y} g\right)_{\lambda}\right|_{H=0}
$$

where $\lambda^{\prime}=b \lambda, b<1$ and $g_{\lambda}=4 \pi^{2} \sigma \lambda^{2-D}$. Here we consider $\lambda \equiv \lambda_{L}$, and $b$ is the momentum shell parameter. From (7) we confirm that the magnetic length scales like the correlation length $L_{c} \propto \Delta^{-v_{1}}$. Since $\left.\partial_{y} \sigma\right|_{y=0} \propto \Delta^{1-\varphi_{1}}$, the crossover exponent in the orthogonal fixed point becomes

$$
\varphi_{1}=2 v_{1}=2 /(D-2)
$$

which was obtained before for 3D [16] and by renormalization of the particle-particle function (Cooper channel) [14].

Near the unitary fixed point at $x=0$ the leading perturbation to the conductivity is $\mathcal{O}\left(x^{2}\right)$. Hence we linearize in $x^{2}$ and deduct the crossover exponent of this perturbation from the scaling behaviour of the correlation function $\partial_{x^{2}} g$ at the unitary fixed point. From equation (4) we obtain

$$
\left.\left(\partial_{x^{2}} g\right)_{\lambda^{\prime}}\right|_{x=0}=\left(1+\left(\frac{0}{g_{\lambda}}+\frac{A}{g_{\lambda}^{2}}\right) \ln b^{2}\right)\left(\partial_{x^{2}} g\right)_{\lambda}
$$

and keeping $\lambda$ fixed we find

$$
\beta_{\partial_{x^{2} g}}=-\frac{\mathrm{d} \ln \left(\partial_{x^{2}} g\right)}{\mathrm{d} \ln b}=-A \varepsilon
$$

where we used the unitary fixed point value $g_{0}^{*}=\sqrt{2 / \varepsilon}, \varepsilon=D-2$, as obtained from the renormalization of (4) at $x=0$ where $\beta_{g}=\varepsilon-2 / g^{2}$. The crossover exponent defined by

$$
\sigma \propto \Delta_{0}^{1 / 2} f_{0}\left(x^{2} / \Delta_{0}^{\varphi_{0}}\right)
$$

follows from $\varphi_{0}=\beta_{\partial_{x^{2} g}} v_{0}$ and $v_{0}=1 /(2 \varepsilon)$ as

$$
\varphi_{0}=\frac{0}{\sqrt{\varepsilon}}-\frac{1}{2} A+\mathcal{O}\left(\varepsilon^{1 / 2}\right) .
$$


Thus the phase invariant fixed point is stable againts the $x^{2}$ perturbation, if $A$ turns out to be positive in a yet to be performed three-loop calculation of $g$. This would then imply that all states are localized in 2D, in particular in the band centre too, up to a critical magnetic field strength at $\omega_{\mathrm{c}} \tau \simeq 1$, where nonperturbative terms lead to a breakdown of perturbation theory [3] with a delocalization effect at the band centre. One may however also, speculate on the existence of a mixed phase with delocalized excitations.

Interaction effects and spin-dependent elastic scattering clearly change the form (3). As a rough estimate the inelastic scattering rate coming from the cooperon mass can be added to $\lambda^{2}$ in all cooperon generated logarithms of (3), i.e. those which are finite for $x \neq 1$. While Zeeman spin splitting is an important cutoff in the particle-particle channel, it is not expected to be relevant for the conductivity as long as up- and down-spin bands are decoupled. For models with a constant density of states only a degeneracy factor 2 appears in $\sigma_{0}$ of equation (3). In a narrow band model however a band centre calculation like (3) cannot apply to both spin bands. A generalization to $E_{\mathrm{F}}$ arbitrarily placed within the subbands only requires the replacements $g_{0} \rightarrow g_{0} \sin \psi$ and $x \rightarrow x \sin \psi$, where $\sin \psi=\rho\left(E_{\mathrm{F}}\right) / \rho(0)$, in equation (4). Indeed $x \sin \psi$ is the new effective coupling of the perturbation introduced by the presence of cooperons.

\section{Acknowledgments.}

I am grateful for discussions with F. Wegner, K. Ziegler, and T. Streit who explained his zeroth Landau level analysis to me. I am particularly indebted for intensive discussions with Boris Shapiro who helped to revise earlier statements during my stay at the Technion in Haifa. This work was supported by the SFB 123 of the DFG and partially by the National Science Foundation under Grant No. PHY77-27084 during the initial stages at the ITP of UC Santa Barbara.

\section{References}

[1] YA AZBel, M., preprint.

[2] Schweitzer, L., Kramer, B. and MacKinnon, A., J. Phys. C 17 (1984) 4111.

[3] Levine, H., Libby, S. B. and Pruisken, A. M., Phys. Rev. Lett. 51 (1983) 1915.

[4] Tinkham, M., Introduction to Superconductivity (McGraw Hill) 1975.

[5] Abrahams, E., Anderson, P. W., Licciardello, D. C. and Ramakrishnan, T. V., Phys. Rev. Lett. 42 (1979) 673.

[6] Oppermann, R. and Wegner, F., Z. Phys. B 34 (1979) 327.

[7] JÜngling, K. and Oppermann, R., Z. Phys. B 38 (1980) 93.

[8] Streit, T., J. Physique Lett. 45 (1984) L-713.

[9] Hikami, S., preprint.

[10] Hikami, S., Larkin, A. I. and Nagaoka, Y., Prog. Theor. Phys. 63 (1980) 707.

[11] Abrahams, E., Anderson, P. W., Lee, P. A. and Ramakrishnan, T. V., Phys. Rev. 24 (1981) 6783.

[12] SCHÄFER, L. and Wegner, F., Z. Phys. B 38 (1980) 113.

[13] Brézin, E., Hikami, S. and Zinn-Justin, J., Nucl. Phys. B 165 (1980) 528.

[14] OppermanN, R. and Ziegler, K., Phys. Rev. B (to be published).

[15] DiCastro, C. and Jona Lasinio, G., in Phase Transitions and Critical Phenomena Vol. 6, eds. C. Domb and. M. S. Green (Acad. Press).

[16] KhmelnitskiI, D. E. and LaRkin, A. I., Solid State Commun. 39 (1981) 1069. 